\title{
Extracorporeal support, during and after lung transplantation: the history of an idea
}

\author{
Fabio Ius ${ }^{1}$, Igor Tudorache ${ }^{1}$, Gregor Warnecke ${ }^{1,2}$ \\ ${ }^{1}$ Department of Cardiothoracic, Transplant and Vascular Surgery, Hannover Medical School, Hannover, Germany; ${ }^{2}$ German Center for Lung \\ Research (DZL/BREATH), Hannover, Germany \\ Contributions: (I) Conception and design: F Ius, G Warnecke; (II) Administrative support: I Tudorache, G Warnecke; (III) Provision of study \\ materials or patients: None; (IV) Collection and assembly of data: F Ius; (V) Data analysis and interpretation: All authors; (VI) Manuscript writing: \\ All authors; (VII) Final approval of manuscript: All authors. \\ Correspondence to: Fabio Ius, MD. Department of Cardiothoracic, Transplant and Vascular Surgery, Hannover Medical School, Carl-Neuberg Strasse \\ 1, 30625 Hannover, Germany. Email: ius.fabio@mh-hannover.de; ius.r@libero.it.
}

\begin{abstract}
During recent years, continuous technological innovation has provoked an increase of extracorporeal life support (ECLS) use for perioperative cardiopulmonary support in lung transplantation. Initial results were disappointing, due to ECLS-specific complications and high surgical risk of the supported patients. However, the combination of improved patient management, multidisciplinary team work and standardization of ECLS protocols has recently yielded excellent results in several case series from highvolume transplant centres. Therein, it was demonstrated that, although the prevalence of complications remains higher in supported patients, there may be no difference in long-term graft function between supported and non-supported patients. These results are important, because most of the patients who require ECLS support in lung transplantation are young and have no other chance to survive, but to be transplanted. Moreover, there is no device for "bridging to destination" therapy in lung transplantation. Of note, the evidence in favour of ECLS support in lung transplantation was never validated by randomized controlled trials, but by everyday experience at the patient bed-side. Here, we review the state-of-the-art ECLS evidence for intraoperative and postoperative cardiopulmonary support in lung transplantation.
\end{abstract}

Keywords: Extracorporeal life support (ECLS); lung transplantation; outcomes

Submitted Jun 30, 2018. Accepted for publication Jul 08, 2018.

doi: $10.21037 /$ jtd.2018.07.43

View this article at: http://dx.doi.org/10.21037/jtd.2018.07.43

\section{Introduction}

Experience is one of the cornerstones of the success of extracorporeal life support (ECLS) in lung transplantation (Figure 1). Over the last 20 years, from the initial case reports on ECLS as rescue before and after lung transplantation $(1,2)$, experience and self-confidence with this technique has grown so much that it is impossible to think of lung transplantation without ECLS nowadays.

The integration of multidisciplinary team work (ECLS team) with the development of standardized management protocols and new technologies has yielded the good results of modern ECLS support (Figure 1), that have recently been published (3-15). Thus, ECLS use has broadened from pretransplant bridging and post-transplant rescue therapy, to elective postoperative extension of intraoperative ECLS support in patients with idiopathic pulmonary arterial hypertension (16-19). While these indications have not been validated by randomized trials, the observational experience of each transplant center helped with refining the indications of ECLS.

Yet, the way was not paved only by successes. A more liberal use of ECLS support has been often criticized, because perioperative results were worse in patients supported by ECLS than in non-supported patients. While, arguably, these failures most likely were due to the 


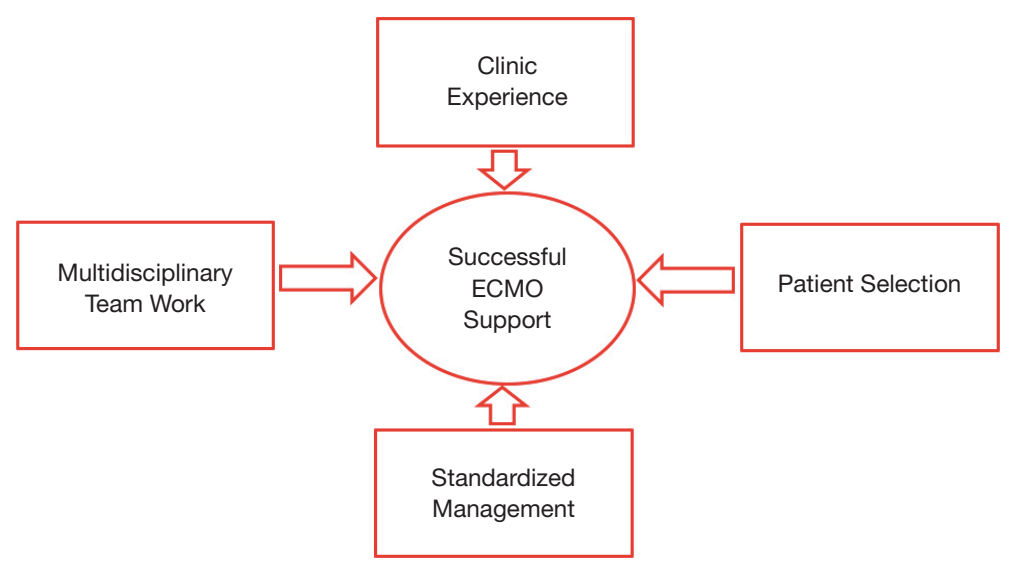

Figure 1 The cornerstones of a successful ECLS program. ECLS, extracorporeal life support.

underlying conditions of the patients, ECLS use is not void of complications, that often are provoked by the need of anticoagulation and arterial and venous vascular accesses. However, increasing expertise and refinements have steadily reduced the prevalence of such complications.

In this chapter, we report the current state-of the-art of ECLS support in patients undergoing lung transplantation. In particular, we focus on the intraoperative and posttransplant use of extracorporeal membrane oxygenation (ECMO), which is by far the most used ECLS support in lung transplantation, and hint briefly at the other support techniques and at ECMO use as a bridge to transplantation (BTT). A more thorough discussion on ECMO use as BTT has been recently reported elsewhere (19). At the end of this review, we present shortly our up-to-date experience with intraoperative and postoperative ECMO in lung transplantation.

\section{ECLS: general considerations}

Over recent years, ECLS technology has been steadily evolving, in order to cope with the requirements of less invasiveness, less prevalence of complications and of leaving supported patients awake and spontaneously breathing $(20,21)$.

However, the basic setup of ECLS systems has remained practically unchanged. Since the inception of the Novalung (Novalung GmbH, Hechingen, Germany) polypentene fibre oxygenator in 2004 (20), the blood of the patient is usually drained by means of an outflow line to the ECLS system and is then pumped back to the patient through an inflow line, after oxygenation and decarboxylation. The blood can be pumped by an external roller or centrifugal pump. Pumpless systems, driven by the pressure gradient between the femoral artery and vein, were en vogue for a number of years, but are less used nowadays, mostly for their inefficacy in oxygenation. A gas exchanger, usually a hollow-fibre oxygenator, is put in series and distally to the pump. Oxygenation depends on blood flow and $\mathrm{FiO}_{2}$, and decarboxylation on sweep gas flow. There are no blood reservoirs and additional suction lines, as in the cardiopulmonary bypass (CPB) that is routinely used in open heart surgery. Therefore, ECLS systems are closed systems, sparing any air-blood interfaces and greatly reducing the surface area of the system, but they are particular susceptible to air embolism.

Figure 2 presents an overview of the ECLS systems that are used in lung transplantation. Different classifications have been proposed. ECLS can be used for pre-transplant, intraoperative or post-transplant support. In case of preand post-transplant support, ECLS may bridge patients to recovery or transplantation/re-transplantation. ECLS can support only the respiratory function (oxygenation/ decarboxylation), or both the respiratory and cardiocirculatory functions. ECLS can be implanted using peripheral venous and arterial accesses, usually via the femoral vein and artery, or a central access, using the right atrium or the pulmonary artery for outflow and the aorta or left atrium for inflow (22).

\section{ECMO: general considerations}

ECMO is by far the most used ECLS system in lung transplantation. Other systems $(20,22-27)$ such as the 


\begin{tabular}{|c|c|c|c|c|}
\hline Time & ECLS Type & Oxygenation & Decarboxylation & Cardiocirculatory support \\
\hline \multirow[t]{8}{*}{ Pretransplant } & Peripheral veno-arterial ECMO & Peripheral organs & Yes & Yes \\
\hline & Central veno-arterial ECMO & Central organs & Yes & Yes \\
\hline & Veno-venous ECMO & Central organs & Yes & No \\
\hline & Veno-venous-arterial ECMO & Central organs & Yes & Yes \\
\hline & $\mathrm{ECCO}_{2} \mathrm{R}$ & No & Partial & No \\
\hline & Peripheral Novalung & No & Yes & No \\
\hline & PA-LA Novalung & Yes & Yes & $\mathrm{RV}$ remodeling \\
\hline & RA-PA Bypass & Yes & Yes & RV support \\
\hline \multirow[t]{3}{*}{ Intraoperative } & Peripheral veno-arterial ECMO & Peripheral organs & Yes & Yes \\
\hline & Central veno-arterial ECMO & Central organs & Yes & Yes \\
\hline & Cardiopulmonary bypass (CPB) & Central organs & Yes & Yes \\
\hline \multirow[t]{4}{*}{ Post-transplant } & Peripheral veno-arterial ECMO & Peripheral organs & Yes & Yes \\
\hline & Central veno-arterial ECMO & Central organs & Yes & Yes \\
\hline & Veno-venous ECMO & Central organs & Yes & No \\
\hline & Veno-venous-arterial ECMO & Central organs & Yes & Yes \\
\hline
\end{tabular}

Figure 2 An overview of the available ECLS systems in lung transplantation. Cardiopulmonary bypass is also included. ECLS, extracorporeal life support.

extracorporeal carbon dioxide removal $\left(\mathrm{ECOO}_{2} \mathrm{R}\right)$ and the peripheral or central Novalung (Novalung GmbH, Hechingen, Germany) have been less frequently used, and usually for bridging to transplantation. The pulmonary artery-left atrium (PA-LA) central Novalung requires a full sternotomy for implant. The peripheral Novalung requires cannulation of the femoral vessels and allows only decarboxylation. Both Novalung system depend on the cardiac function of the patient for pumping blood through the system. The $\mathrm{ECOO}_{2} \mathrm{R}$ allows only a partial decarboxylation.

On the contrary, ECMO allows the complete spectrum of support in lung transplantation. Its versatility allows for pre-transplant support, which, in comparison with other ECLS systems, can be directly continued intraoperatively and post-transplant (Figure 2).

\section{Veno-venous ECMO}

The veno-venous ECMO modality allows only for respiratory support. A combination of veno-venous ECMO and atrial septostomy may allow for cardiocirculatory support too, but its application has been limited by the difficulty to correctly size the septal defect, the tendency of the defect to shrink over time and the necessity to close the defect during ECMO weaning $(20,28)$.

A dual or one site cannulation strategy can be used. In the case of a dual-site strategy, which is the preferred at our Institution, the outflow and inflow cannulas are usually placed percutaneously using the Seldinger technique in the femoral and internal jugular veins, respectively. This strategy allows for some degree of patient mobilisation but it does not allow for patient ambulation. Yet, the main advantage over the single cannula/double lumen strategy is the higher maximum ECMO blood flow, allowing for better oxygenation. In the case of one site cannulation strategy, a dual lumen cannula (Avalon Elite, Maquet, Rastatt, Germany) is placed percutaneously in the internal jugular vein or in the subclavian vein, under transoesophageal echocardiographic control. This strategy is more suitable, if an awake bridge to transplantation (BTT) strategy is planned, since it allows for patient ambulation. However, 
cannula dislocation must be avoided during patient mobilisation. The veno-venous ECMO has been preferred for BTT and for bridging to recovery those patients who developed severe primary graft dysfunction (PGD) after transplantation (29). However, patients with lung fibrosis and secondary pulmonary arterial hypertension and transplanted patients with PGD and severe haemodynamic compromise may require peripheral or central veno-arterial ECMO (30).

\section{Veno-arterial ECMO}

Veno-arterial ECMO can be used for bridging patients with prevalent cardiocirculatory failure to lung transplantation, for intraoperative and post-transplant support. In this last case, intraoperative support can prophylactically be extended postoperatively in those patients with idiopathic pulmonary arterial hypertension, in order to avoid the development of severe cardiogenic oedema caused by diastolic dysfunction of the left ventricle (16-18). A peripheral or central cannulation strategy can be used.

In the case of peripheral cannulation, the femoral vein and artery are cannulated, usually percutaneously. In small size patients, such as children and small women, cut down and direct surgical exposition of the vessels are preferred. In babies, the jugular vein and a carotid artery are surgically isolated and cannulated. However, femoral veno-arterial ECMO guarantees oxygenation only of the periphery, because arterial blood flow from the ECMO at best reaches the distal aortic arch. Here, it mixes with blood pumped by the heart and oxygenated by the patient lungs, especially if the cardiac function is preserved. This effect usually does not cause any consequence in patients with idiopathic pulmonary arterial hypertension, where the oxygenatory function of the lung is preserved. On the contrary, if lung function is impaired, as in patients with pulmonary fibrosis, central organs such as the heart and brain are perfused with poorly oxygenated blood (watershed effect, Harlequin Syndrome). Controlling the arterial blood gases from the right radial artery and continuously measuring the peripheral oxygen saturation from the right hand or ear lobes are useful tools for recognizing the development of such syndrome.

Centrally cannulated veno-arterial ECMO might mitigate this problem, since arterial blood can be directly infused into the ascending aorta (31). However, it requires a full sternotomy and hinders patient mobilization and an awake BTT strategy, not the least because additional major surgery is necessary for ECMO explant. Alternatively, the arterial inflow cannula can be placed into the right subclavian artery, through the interposition of a Dacron prosthesis. This configuration ("sport" configuration) allows for patient ambulation and rehabilitation $(4,20)$. Another option is to create a veno-veno-arterial ECMO circuit by adding an arterial inflow cannula into the jugular vein (32), in parallel with the arterial inflow into the femoral artery. In this case, however, particular care must be paid in balancing the flows through the two inflow cannulas, according to the respiratory and cardiocirculatory needs of the patient. As a rule of thumb, 1/3 of the inflow should be dedicated for respiratory support and the remaining for cardiocirculatory support. The use of a flowmeter is essential if this support modality is used.

Careful attention must be paid to the development of ischemia in the leg, where the arterial inflow cannula is placed, especially in BTT patients and in patients with post-transplant veno-arterial ECMO $(22,33,34)$. At our institution, an antegrade leg perfusion cannula is placed distally to the inflow arterial cannula, whenever possible. Moreover, the arterial pulses as well as cannula patency are checked regularly. Near-infrared spectroscopy is used to continuously monitor the oxygen saturation of the leg, where the inflow cannula has been placed.

\section{ECMO implant technique and management}

For each ECMO modality, cannula sizes depend on patient size and blood flow requirements. Cannulation strategy and choice of cannula types depend on institutional preference. Cannula insertion and placement can be guided by sonography and controlled by transoesophageal echocardiography, especially in the case of the dual lumen cannula for veno-venous ECMO. However, these tools are not always available, especially in the emergency setting. At our institution, a chest X-ray is performed to control cannula position after ECMO implant.

The management of anticoagulation at ECMO implant and during ECMO support is of paramount importance to avoid bleeding. Many Institutions have developed their own protocols. At our Institution, we usually infuse a bolus of 5.000 units of unfractionated heparin before cannula insertion and then guide anticoagulation by regularly measuring the activated clotting time (ACT) every 4 hours, as well as the international normalized ratio (INR), the Quick value, the activated partial thromboplastin time (aPTT), the plasma levels of fibrinogen, factor V and II at least twice daily. Heparin is started usually not before 
Table 1 Published case series on ECMO as bridge to transplantation

\begin{tabular}{|c|c|c|c|c|}
\hline Study & $\mathrm{N}$ patients & $\begin{array}{l}\text { Patients bridged } \\
\text { to transplant }\end{array}$ & $\begin{array}{l}\text { Intention-to-treat } \\
\text { survival }\end{array}$ & $\begin{array}{l}\text { Survival after } \\
\text { transplantation }\end{array}$ \\
\hline Bermudez et al., 2011 (35) & 17 & $15(88.2)$ & - & $65 \%$ at 3 years \\
\hline Fuehner et al., 2012 (36) & 26 & $20(76.9)$ & - & $80 \%$ at 6 months \\
\hline Lang et al., 2012 (37) & 38 & $34(89.5)$ & - & $60 \%$ at 1 year \\
\hline Lafarge et al., 2013 (40) & 36 & $30(83.3)$ & $50.4 \%$ at 2 -years & $60.5 \%$ at 2 years \\
\hline Dellgren et al., 2015 (41) & 20 & $16(80.0)$ & $62 \%$ at 1 year & $69 \%$ at 1 year \\
\hline Yeo et al., 2017 (42) & 19 & $14(73.7)$ & $57.9 \%$ & $64.3 \%$ \\
\hline Hoetzenecker et al., 2018 (6) & 71 & $63(88.7)$ & $51 \%$ at 5 years & $60 \%$ at 5 years \\
\hline Hakim et al., 2018 (7) & 30 & $26(86.7)$ & $70 \%$ at 3 years & $80 \%$ at 3 years \\
\hline
\end{tabular}

Values are reported as $\mathrm{n}(\%)$ or $\%$.

48 hours after ECMO implant, aiming at an ACT set at 160-180 s.

The management of ECMO patient before or after lung transplantation will be further discussed in the following paragraphs and it depends on the patient cardiopulmonary conditions and the type of support. Anyway, at our institution, heart rate, central venous pressure, arterial blood pressure, pulmonary arterial pressure, body temperature, and peripheral oxygen saturation are continuously monitored. Antibiotic therapy is empirically performed with flucloxacillin, meropenem and voriconazole, or antibiogram-guided, if there is evidence of any pathogen.

\section{ECMO as a bridge to transplantation}

Table 1 reports the most recent case series on ECMO as BTT. These case series show that survival results have been steadily improving over the last years and that (I) a careful patient selection, (II) the implementation of awake ECMO protocols and (III) center transplant volume and experience were fundamental to this improvement (3-7,35-49).

Patient selection implies ethical issues, because, due to the organ donor shortage, it could be questioned whether organs should be really offered to such high-risk recipients. However, patients selected for undergoing BTT often are younger than other wait list patients. In contrast to patients listed for a heart transplantation, there is no device available for bridge to destination therapy in lung transplantation. Moreover, the most recent case series have even shown that graft survival was similar in patients who were bridged to transplantation with ECMO and those who were not. Todd et al. reported a 1-year survival of $100 \%$ in the bridge to transplant group and of $91 \%$ in the non-bridge to transplant group, with an excellent functional status in both groups (3). Our group has recently shown that overall graft survival did not differ between BTT and non-BTT patients $(79 \%$ vs. $90 \%$ and $61 \%$ vs. $68 \%$ at 1 and 5 years, respectively, $\mathrm{P}=0.13$ ) and that ECMO as BTT did not emerge as risk factor for graft survival at the multivariate analysis (5).

Anyway, each institution has its protocol for selecting candidates for BTT, whose indications and contraindications have been recently summarized by Loor et al. (19). At our institution, we usually avoid considering BTT for patients who showed irreversible end-organ damage or sepsis. Older age remains a relative contraindication. For example, we usually do not proceed to transplanting older patients with pulmonary fibrosis if they require invasive mechanical ventilation while on ECMO. Patients requiring retransplantation are also carefully selected, since results of ECMO as BTT in these 
patients have been less satisfactory $(6,45)$. Moreover, we prefer considering for ECMO as BTT those patients who have already been listed at our institution. Patients without previous transplant evaluation who were transferred to our intensive care unit from a peripheral hospital under mechanical ventilation and ECMO support are considered for transplant only after extubation and careful evaluation of end-organ damage (5).

The introduction of the "awake" strategy as early as 2008 represented an important milestone in the management of pre-transplant patients at our institution (36). Since then, many other case series have validated the benefit of spontaneous breathing and mobilisation during ECMO support, not only before, but also after lung transplantation (3-6,16,17,44,45,48). Moreover, new cannulas and implant techniques have dramatically improved the chance of patients being rehabilitated during ECMO support (50-53). Thereby, the patient muscular deconditioning due to the immobilisation and mechanical ventilation are remarkably reduced. Hayanga et al. have recently demonstrated that extubated patients on ECMO as BTT fare better than intubated patients on ECMO including those patients who required only mechanical ventilation before transplantation (54).

\section{ECMO for intraoperative support}

In 2010, we changed our protocol for intraoperative cardiopulmonary support during lung transplantation, replacing CPB with peripheral veno-arterial ECMO (55). Our decision was based on the following considerations: (I) the versatility of intraoperative ECMO, which allowed for continuing support in those patients who could not be weaned from it; (II) the possibility of performing major cardiac surgery, for example coronary artery bypass grafting (CABG) on beating heart technique with ECMO support; (III) the lower amount of heparin required by ECMO in comparison to CPB; (IV) the lower amount of priming volume in ECMO circuits; (V) the lower degree of systemic inflammatory reaction (SIRS) after ECMO; and (VI) the feasibility of intraoperative ECMO support as previously evidenced by the colleagues from Vienna, Austria (56).

We do not exclude a priori the use of CPB and still use it in the following situations: (I) concomitant need of repairing an intracardiac defect, such as an atrial septal defect, and (II) in case of unexpected massive blood loss, for example when the pulmonary artery is accidentally injured during insolation for clamping. Regarding the first point, we perform the intracardiac repair under $\mathrm{CPB}$, wean it and then proceed to lung transplantation without $\mathrm{CPB}$ support.

In a recent publication (12), we have divided patients undergoing intraoperative ECMO support in two categories, those requiring an a-priori ECMO support and those without a-priori indication for ECMO. A priori ECMO was reserved for the following patients: (I) patients already bearing ECMO as BTT; (II) patients with idiopathic pulmonary arterial hypertension as indication to transplantation or secondary supra-systemic pulmonary hypertension; (III) patients where a lobar transplantation was planned in order to avoid hyper-perfusion of the transplanted lobe during single lung ventilation; and (IV) patients requiring concomitant CABG. In these patients, it would not be possible to perform transplantation safely without ECMO support. Strikingly, we demonstrated that survival did not differ between patients who required intraoperative ECMO and those who did not, and that the intraoperative use of ECMO did not emerge as a risk factor for in-hospital mortality or mortality after hospital discharge (12).

Since 2010, several case series and meta-analyses have demonstrated the superiority of ECMO vs. CPB for intraoperative support and better outcomes in lung transplantation (Table 2) $(8-11,13,14,57-60)$. Some authors have recently proposed the routine use of intraoperative ECMO in lung transplantation, in order to allow controlled perfusion and protective ventilation of the graft during transplantation and thus reduce the risk of later PGD $(14,57)$.

These suggestions should be regarded with caution, however, given that ECMO therapy is associated with ECMO-specific complications, such as bleeding and vascular complications $(33,34,61)$, which might impair perioperative results. Central cannulation of the aorta and right atrium has been proposed as a strategy to reduce vascular complications (31). However, central cannulation often requires a clamshell incision and, in those patients requiring post-transplant continued ECMO support, later switch to peripheral ECMO.

In our opinion, the identification of those patients who really need ECMO support and the avoiding of ECMO implant under urgent/emergent conditions, for example during or after pneumonectomy, may help with reducing ECMO-specific complications. Therefore, we have previously designed a decision algorithm that helps with identifying those patients who are at risk of requiring intraoperative ECMO support (12). In particular, before starting the operation, it is important to check the 
Table 2 Published case series on ECMO for intraoperative support during lung transplantation

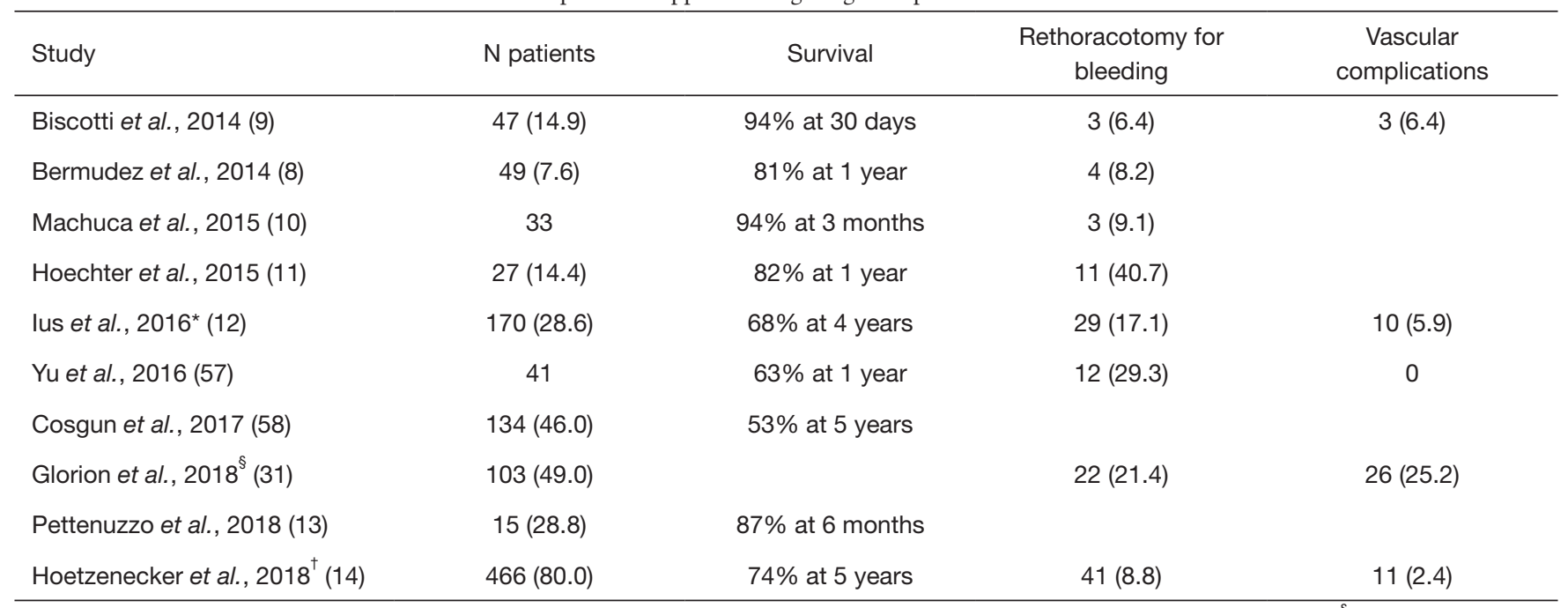

Values are reported as $\mathrm{n}(\%) .{ }^{*}, 95$ patients with a priori ECMO implant, 75 patients without a priori ECMO implant. ${ }^{\S}, 49$ patients with peripheral veno-arterial ECMO and 54 patients with central veno-arterial ECMO. ${ }^{\dagger}, 343$ patients with intraoperative ECMO support only, 123 patients with prolonged postoperative ECMO support.

echocardiographic reports, the results of the right heart catheterization and the $\mathrm{X}$-ray of the recipient. The presence of a secondary pulmonary arterial hypertension, of a dilated and hypertrophied right ventricle, and of small thoracic cavities at the chest X-ray should alert the surgeon to the higher risk of requiring intraoperative ECMO. Close communication with the retrieval surgeon is of paramount importance. The presence of contusions or chronic atelectasis in one of the lungs should prompt the decision of implanting the better lung first. Before incision, the surgeon should check that nitric oxide (NO) ventilation is switched on. During implantation, the surgeon should closely communicate with the anaesthesiologist, in order to identify possible signs of respiratory and haemodynamic instability and counteract against them quickly. If cardiopulmonary conditions worsen after test clamping of the right or left pulmonary arteries, the surgeon should look for potentially reversible causes, and to proceed with implantation of an ECMO if cardiopulmonary instability persists. Usually, indication for ECMO implant is set if a combination of the following conditions ensues: (I) hypercapnia; (II) decrease of arterial saturation less than $90 \%$; (III) cardiac index less than $2 \mathrm{~L} / \mathrm{min} / \mathrm{m}^{2}$; and (IV) increase of the pulmonary arterial pressure to supra-systemic values. The second evaluation is performed after implanting the first lung, at clamping of second pulmonary artery, checking whether the freshly implanted lung is able to yield adequate oxygenation and accommodate the entire cardiac output (12). In patients at risk, we pre-emptively place guidewires in the right femoral vein and artery to prepare a safe and quick ECMO implant if necessary later.

At the end of transplantation, before closing the thoracotomies, the possibility of explanting the ECMO is evaluated by checking the arterial blood gases and pulmonary arterial pressure at $100 \% \mathrm{FiO}_{2}$. If these parameters are satisfactory, ECMO is explanted. In patients with idiopathic pulmonary arterial hypertension, venoarterial ECMO might be left in place as per institutional protocol. Careful attention is paid to meticulous haemostasis. We suggest antagonizing heparin with halfdose protamine. Recently, Narm et al. have shown that increasing donor age, donor $\mathrm{PaO}_{2}$ and increasing operation duration were independent risk factors of weaning failure from intraoperative ECMO in their experience (62).

A particular mention deserves the possibility of performing concomitant CABG and lung transplantation, as a beating heart technique and under ECMO support. Indeed, it is not always possible to treat the coronary artery disease (CAD) with PTCA and stenting before transplantation, especially if a complex stenosis or a twovessel CAD are present. At our institution, we perform lung transplantation and CABG using a clamshell incision. We usually perform first the lung transplantation and then the CABG. ECMO can be implanted at the beginning of 
Table 3 Published case series on secondary ECMO implant after lung transplantation

\begin{tabular}{|c|c|c|c|c|}
\hline Study & $\mathrm{N}$ patients & Survival & Weaned patients & Time ECMO-weaning (days) \\
\hline Dahlberg et al., 2004 (65) & $16(9.3)$ & $46 \%$ at 2 years & - & - \\
\hline Oto et al., 2004 (66) & $10(2.1)$ & - & $4(40.0)$ & 4 (mean) \\
\hline Mason et al., 2006 (67) & $22(4.0)$ & $41 \%$ at 1 year $^{\dagger}$ & - & 4 (median) \\
\hline Bermudez et al., 2009 (69) & $58(7.6)$ & $40 \%$ at 1 year & $39(67.2)$ & 5.5 (mean) \\
\hline Hartwig et al., 2012 (15) & $28(6.0)$ & $64 \%$ at 1 year & $27(96.4)$ & 3.6 (mean) \\
\hline Marasco et al., 2012 (70) & 24 & $25 \%$ at hospital discharge & $14(58.3)$ & 4.5 (median) \\
\hline Mulvihill et al., 2018 (71) & $107(5.1)$ & $62 \%$ at 6 months & - & - \\
\hline
\end{tabular}

Values are reported as $\mathrm{n}(\%){ }^{\dagger}$, survival was $41 \%$ at 1 year in patients with early graft failure and acute rejection and only $3 \%$ in patients with sepsis and pneumonia.

transplantation or at its end, before CABG. Since both the internal mammary arteries are sacrificed during the clamshell incision, saphenous vein grafts are usually used. The Octopus system (Medtronic Inc, Minn., MN, USA) might be used for stabilizing the coronary artery during anastomosis. ECMO is usually explanted at the end of CABG.

\section{ECMO for postoperative support}

The development of severe graft dysfunction after lung transplantation is a dramatic event and it sometimes requires secondary ECMO therapy for graft rescue, if conservative therapy fails. Graft function and survival were worse in patients who required secondary ECMO than in patients who did not require it $(15,63-71)$ (Table 3). However, early recognition of incoming graft dysfunction with prompt ECMO implant has partially improved the prognosis of these complications (15).

Severe graft dysfunction is mainly due to the development of primary graft dysfunction early after transplantation, and due to acute rejection or pneumonia later after transplantation. Mason et al. (67) and Marasco et al. (70) have shown that secondary ECMO for early causes of graft dysfunction yielded better weaning and survival results than secondary ECMO for later causes. This finding shows that ECMO therapy for severe PGD should not be limited to a mere rescuing role but should be considered pre-emptively in those patients who are at particular higher risk of developing it $(72,73)$, such as those transplanted for idiopathic pulmonary arterial hypertension.
In these patients, PGD was supposed to be a consequence of endothelial injury from shear-stress forces applied by a well-trained right ventricle resulting in subsequent pulmonary oedema. However, we and others have recently demonstrated that the primary mechanism causing PGD might be not right ventricle-related, but more likely due to diastolic dysfunction of the left ventricle, and should rather be called cardiogenic oedema. In fact, the long-standing underfilling of the left ventricle in the presence of reduced cardiac output secondary to very high pulmonary vascular resistance may result in deconditioning of the left ventricle, rendering the left ventricle incapable of handling a normal preload in the early postoperative period (16). The characteristic pulmonary oedema of PGD is a consequence of a sudden increase of the left ventricular end diastolic pressure and of the left atrial pressures soon after graft reperfusion, aggravated further after extubation of the patient. The postoperative use of veno-arterial ECMO provides time for gradual adaption of the left ventricle to the new haemodynamic situation after transplantation. The importance of the left ventricular diastolic dysfunction in causing PGD has been recently demonstrated by Porteous et al. (74). They suggested that veno-arterial ECMO may allow for controlled filling and recovery of the left ventricle, preventing acute increases in pulmonary venous pressure in the early period after lung transplantation.

Therefore, at our institution, patients with idiopathic pulmonary arterial hypertension are usually transplanted under veno-arterial ECMO support, which is not weaned at the end of transplantation, but continued directly 
in the postoperative period. Full ECMO support is maintained for at least 5 days. In the meanwhile, patients are extubated (awake ECMO) and undergo rehabilitation, whenever possible. Cardiac function is regularly checked with transthoracic echocardiography. Pulmonary arterial and left atrial pressures are continuously monitored using a pulmonary arterial catheter and an additional pressure line, that was previously placed in the left atrium during transplantation, respectively. Beyond day 5 after transplantation, ECMO weaning is initiated while controlling for left ventricular function during intermittent reduction of ECMO blood flow to $0.6 \mathrm{~L} / \mathrm{m}^{2}$ body surface area. When there are neither echocardiographic signs of left ventricular diastolic dysfunction nor increases of pressure in left atrium over $10 \mathrm{mmHg}$ during reduction of blood flow, the ECMO flow is reduced in $0.5 \mathrm{~L}$ per minute steps, until a flow of $0.6 \mathrm{~L} / \mathrm{m}^{2}$ body surface area is reached and then the ECMO is explanted (16). During ECMO reduction, a negative fluid balance is carefully maintained. Moreover, we keep the arterial blood pressure under a mean of $80 \mathrm{mmHg}$ using beta-blockers, which are usually continued after ECMO explant, and avoid the use of any inotropic drugs, since left and right systolic ventricular function is usually preserved in these patients. We have recently published our complete surgical experience in patients with severe pulmonary arterial hypertension treated with this ECMO protocol (17). We showed that survival did not differ between patients transplanted for severe pulmonary hypertension and those transplanted for other underlying conditions and that a normal left ventricular diastolic function was achieved at last echocardiographic control. In patients with severe pulmonary hypertension, 5-year survival was $70 \%$, which was comparable with the 5 -year survival of patients transplanted for other conditions (69\%).

Excellent results using prolonged veno-arterial ECMO support in patients with idiopathic pulmonary arterial hypertension have been recently published by the Vienna group (18). They started using veno-arterial ECMO for idiopathic pulmonary arterial hypertension as early as 2000 and reported a 5 -year survival of $87.4 \%$. Yet, the management protocol is different. Intraoperatively, they used centrally implanted veno-arterial ECMO, which was switched to peripherally implanted ECMO at the end of transplantation. They kept patients on veno-arterial ECMO support for a shorter period of time (median of 2.5 days) after transplantation and did not proceed to extubation before ECMO weaning. After ECMO weaning, they prefer ventilating patients with a protective low tidal volume pattern and address aggressively the normalization of fluid balance, which explains the higher prevalence of hemodialysis treatment in their experience.

Considering the Hannover and Vienna experience together, we suggest that patients with severe pulmonary arterial hypertension who were under prophylactic postoperative veno-arterial ECMO support should not be considered anymore as having automatically PGD 3 scores, as reported by the most recent consensus statement on PGD (72).

Finally, although other recipient and donor risk factors for PGD have been reported (73), we apply our ECMO protocol only in patients with severe pulmonary hypertension. We carefully monitor the other patients, in order to detect the first signs of PGD as soon as possible.

\section{Hannover experience with intraoperative ECMO}

Before concluding, we present briefly our recent experience with ECMO in lung transplantation. Results are reported in Tables 4-7, and in Figures 3,4. Data were reported as $\mathrm{n}(\%)$ and median (interquartile range, IQR), for categorical and continuous variables, respectively.

Between January 2010 and May 2018, 1,042 patients underwent lung transplantation at our institution. Among these patients, $22(2 \%)$ patients were transplanted with $\mathrm{CPB}$ support and were thus excluded from the analysis.

Among the remaining 1,020 (98\%) included patients, 281 $(28 \%)$ required intraoperative veno-venous or veno-arterial ECMO support, and 739 (72\%) patients did not. Follow-up ended in June 2018, was 100\% complete and amounted to a median of 37 (IQR, 17-61) months.

ECMO patients showed a higher surgical risk than no ECMO patients, with a higher prevalence of pulmonary fibrosis and idiopathic pulmonary arterial hypertension as indication to transplantation. ECMO patients were younger than no ECMO patients and were more often females. Of note, donor characteristics, except for female sex, did not differ between ECMO and no ECMO patients.

Postoperatively, prevalence of major complications was higher in ECMO than no ECMO patients, whose consequence was a higher in-hospital mortality in ECMO than no ECMO patients. Although overall survival was worse in ECMO patients, survival did not substantially differ among groups at 5 years (Table 7, Figure 4). Moreover, survival conditioned to hospital discharge and freedom from chronic lung allograft dysfunction (CLAD) did not differ between groups over the whole study period. 
Table 4 Preoperative recipient data in patients who required ECMO support during transplantation and in patients who did not

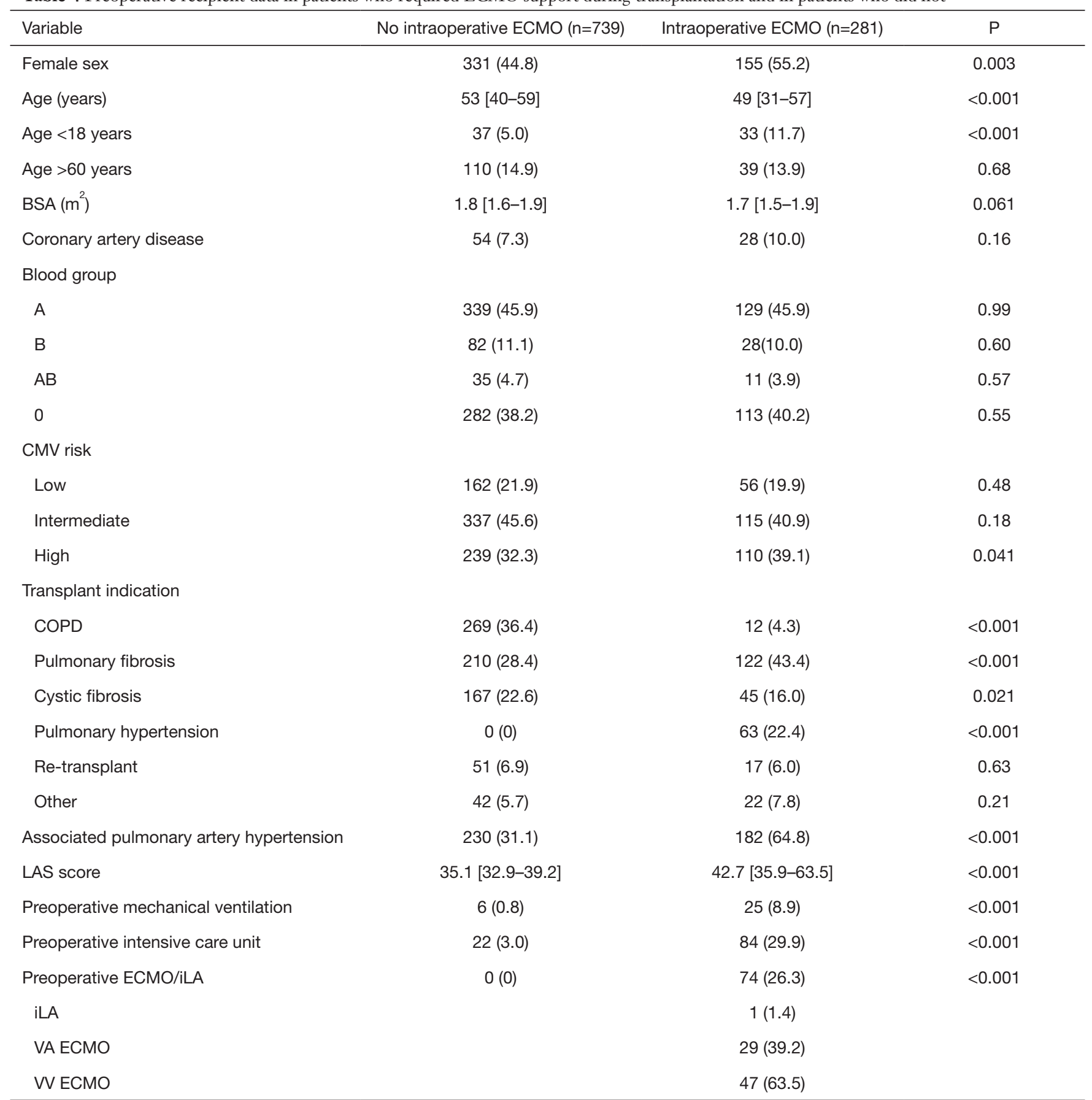

Values are expressed as median [IQR, interquartile range] or $\mathrm{N}$ of patients (\%). BSA, body surface area; CMV, cytomegalovirus; COPD, chronic obstructive pulmonary disease; ECMO, extracorporeal membrane oxygenation; iLA, interventional Lung Assist Novalung; LAS, lung allocating score; VA, veno-arterial; VV, veno-venous. 
Table 5 Donor and intraoperative recipient characteristics in patients who required ECMO support during transplantation and in patients who did not

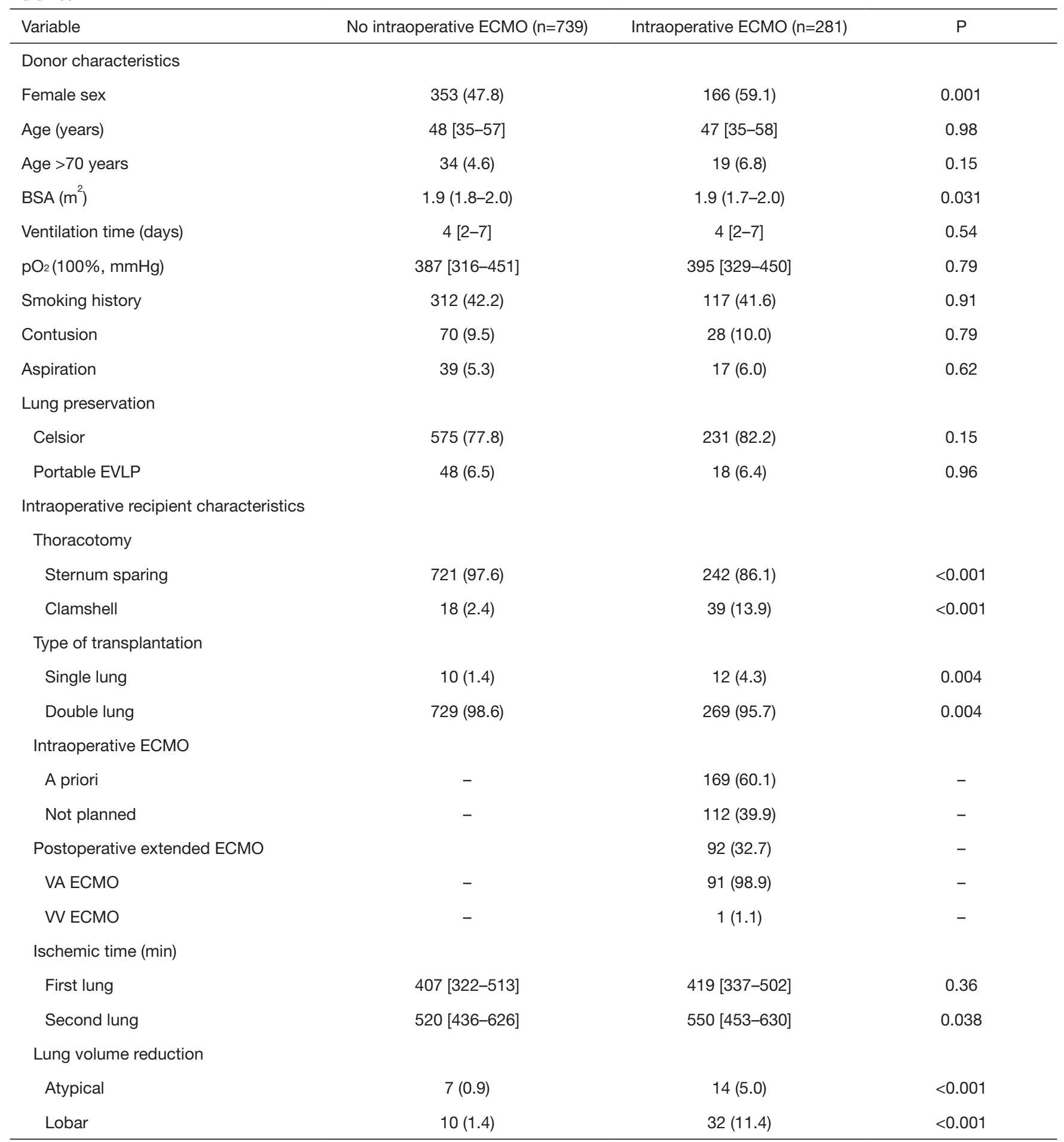

Values are expressed as median [IQR, interquartile range] or $\mathrm{N}$ of patients (\%). BSA, body surface area; ECMO, extracorporeal membrane oxygenation; EVLP, ex-vivo lung perfusion; VA, veno-arterial; VV, veno-venous. 
Table 6 Postoperative data in patients who required ECMO support during transplantation and in patients who did not

\begin{tabular}{|c|c|c|c|}
\hline Variable & No intraoperative ECMO ( $n=739)$ & Intraoperative ECMO $(n=281)$ & $\mathrm{P}$ \\
\hline 24 hours & $72(9.7)$ & $88(31.3)$ & $<0.001$ \\
\hline 48 hours & $66(8.9)$ & $99(35.2)$ & $<0.001$ \\
\hline 72 hours & $47(6.4)$ & $81(28.8)$ & $<0.001$ \\
\hline New dialysis & $27(3.7)$ & $56(19.9)$ & $<0.001$ \\
\hline Atrial fibrillation & $69(9.3)$ & $50(17.8)$ & $<0.001$ \\
\hline Vascular complications & $2(0.3)$ & $27(9.6)$ & $<0.001$ \\
\hline Cerebrovascular disorder & $10(1.4)$ & $5(1.8)$ & 0.57 \\
\hline PRBCs (units) & $5[3-8]$ & $12[7-26]$ & $<0.001$ \\
\hline PC (units) & $0[0-2]$ & $2[2-7]$ & $<0.001$ \\
\hline FFP (units) & $4[3-6]$ & $9[5-16]$ & $<0.001$ \\
\hline Secondary ECMO & $5(0.7)$ & $15(5.3)$ & $<0.001$ \\
\hline VA ECMO & $4(0.5)$ & $11(3.9)$ & \\
\hline VV ECMO & $1(0.1)$ & $5(1.8)$ & \\
\hline Tracheostomy & $45(6.1)$ & $72(25.6)$ & $<0.001$ \\
\hline Ventilation time, hours & $1[1-1]$ & $2[1-4]$ & $<0.001$ \\
\hline Tacrolimus & 518 (71.9) & $179(72.5)$ & 0.87 \\
\hline \multicolumn{4}{|c|}{ Immunosuppressive therapy at last outpatient control $^{\dagger}$} \\
\hline Cyclosporine & $159(22.1)$ & 45 (18.2) & 0.19 \\
\hline Tacrolimus & 555 (77.1) & $198(80.2)$ & 0.31 \\
\hline
\end{tabular}

Values are expressed as median [IQR, interquartile range] or $\mathrm{N}$ of patients $(\%){ }^{\dagger}$, in-hospital deaths $(\mathrm{n}=53)$ are excluded. FFP, fresh frozen plasma; ICU, intensive care unit; PC, platelet concentrate; PGD, primary graft dysfunction; PRBCs, packed red blood cells; VA, venoarterial; VV, veno-venous. 
Table 7 Outcomes in patients who required ECMO support during transplantation and in patients who did not

\begin{tabular}{|c|c|c|c|}
\hline Variable & No intraoperative ECMO $(n=739)$ & Intraoperative ECMO (n=281) & $\mathrm{P}$ \\
\hline 3 years & $84 \pm 2$ & $74 \pm 3$ & \\
\hline 5 years & $72 \pm 2$ & $68 \pm 3$ & \\
\hline Patient conditioned to hospital discharge (\%) & & & 0.50 \\
\hline 5 years & $74 \pm 2$ & $78 \pm 3$ & \\
\hline Graft survival (\%) & & & 0.031 \\
\hline 3 years & $82 \pm 2$ & $73 \pm 3$ & \\
\hline 5 years & $69 \pm 2$ & $64 \pm 3$ & \\
\hline Infection & $20(2.8)$ & $9(3.6)$ & 0.49 \\
\hline Malignancy & $17(2.4)$ & $4(1.6)$ & 0.49 \\
\hline Cardiac & $13(1.8)$ & $3(1.2)$ & 0.77 \\
\hline Other & $14(1.9)$ & $6(2.4)$ & 0.64 \\
\hline Freedom from biopsy-confirmed rejection (\%) & & & 0.71 \\
\hline 1 year & $65 \pm 2$ & $61 \pm 4$ & \\
\hline 3 years & $56 \pm 2$ & $52 \pm 4$ & \\
\hline 5 years & $52 \pm 2$ & $51 \pm 4$ & \\
\hline 1 year & $54 \pm 2$ & $55 \pm 3$ & \\
\hline 3 years & $41 \pm 2$ & $39 \pm 3$ & \\
\hline 5 years & $37 \pm 2$ & $34 \pm 4$ & \\
\hline Freedom from CLAD (\%) & & & 0.98 \\
\hline 3 years & $77 \pm 2$ & $75 \pm 3$ & \\
\hline 5 years & $67 \pm 2$ & $66 \pm 4$ & \\
\hline
\end{tabular}

Values are expressed as mean \pm SD (\%) or $\mathrm{N}$ of patients (\%). ${ }^{\dagger}$, patients who died before hospital discharge $(\mathrm{n}=53)$ were not considered, Intraoperative ECMO $n=247$, No intraoperative ECMO $n=720$. *, No intraoperative ECMO $n=658$, Intraoperative ECMO $n=199$. CLAD, chronic lung allograft dysfunction; ISHLT, International Society of Heart and Lung Transplantation. 
A

\% ECMO as a bridge to transplantation

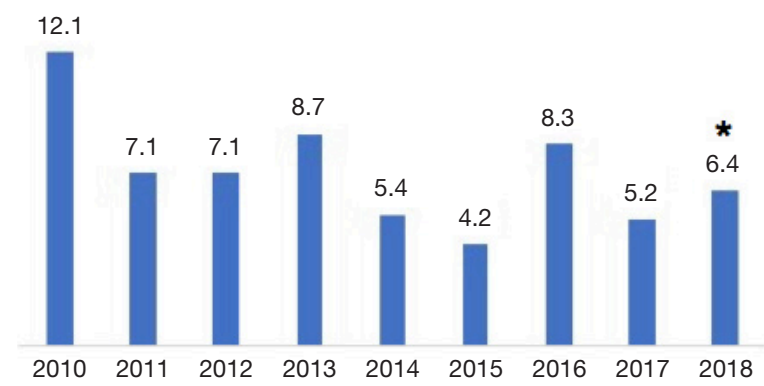

C

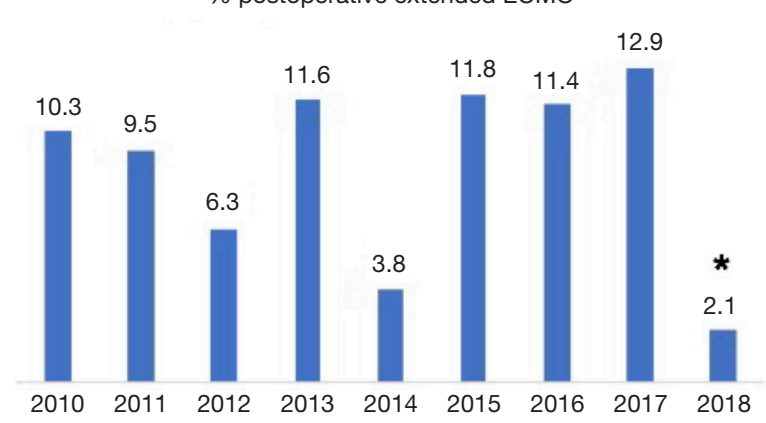

B

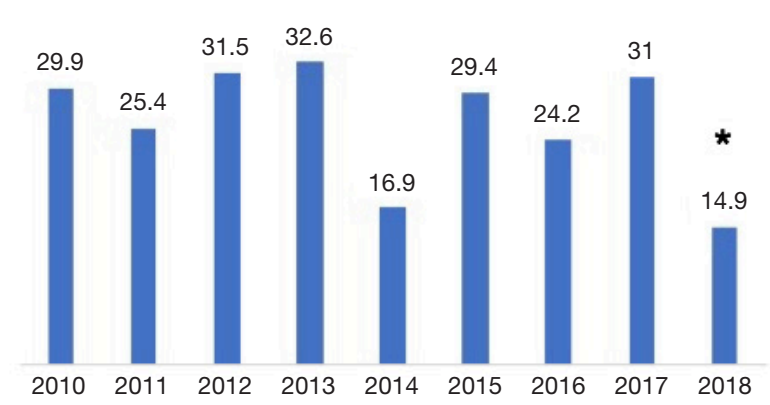

$\mathrm{D}$

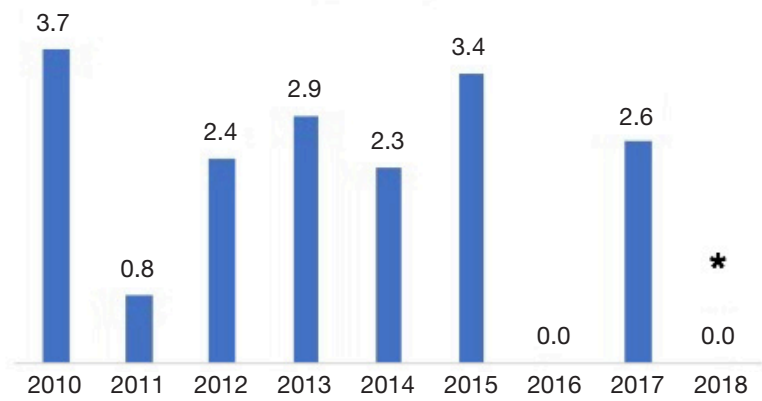

Figure 3 The figure shows the percentage of: (A) patients bridged to lung transplantation with ECMO per each study year at our institution; (B) patients requiring intraoperative ECMO support; (C) patients requiring extended postoperative ECMO support; and (D) patients requiring secondary ECMO support after transplantation. At our institution, 107 patients were transplanted in 2010, 126 in 2011, 127 in 2012, 138 in 2013, 130 in 2014, 119 in 2015, 132 in 2016, 116 in 2017, and 47 until May 2018.

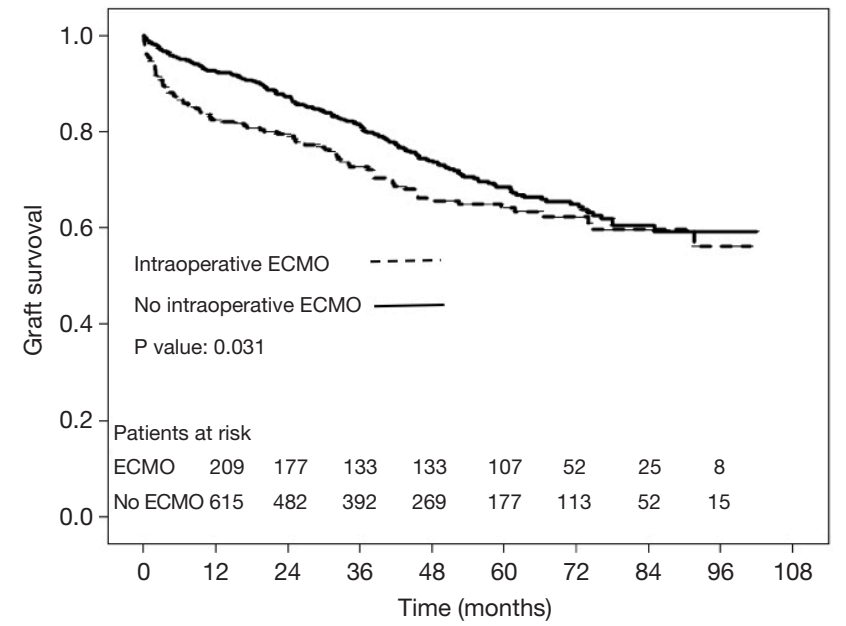

Figure 4 The graft survival (intended as freedom from mortality and retransplantation) in patients who required ECMO support during lung transplantation $v$ s. patients who did not require it, at our institution. Patients at risk are reported above the $\mathrm{X}$ axis.
On the contrary, median values of forced expiratory volume in 1 second (FEV1, \% predicted) at 1 year and at last control (performed at median of 35 months after transplantation) were significantly lower in ECMO than in no ECMO patients (75 vs. 90, $\mathrm{P}<0.001$; and 66 vs. 77, $\mathrm{P}<0.001$, respectively). However, this finding can be also explained by the higher prevalence of females in the ECMO group.

\section{Conclusions}

The introduction of ECLS for cardiopulmonary support in lung transplantation has revolutionized the management of transplanted patients. It has allowed for an expansion of the transplant indications, and has allowed for transplanting patients who were inexorably condemned to a certain death before its introduction.

This revolution was not validated by randomized 
controlled trials, but by the everyday experience at the patient bed-side.

Further progress in ambulation of ECMO patients, in the reduction of complications, and eventually even in full implantability of the devices are expected and will revolutionize the field of lung transplantation for the years to come.

\section{Acknowledgements}

None.

\section{Footnote}

Conflicts of Interest: The authors have no conflicts of interest to declare.

\section{References}

1. Jurmann MJ, Haverich A, Demertzis S, et al. Extracorporeal membrane oxygenation as a bridge to lung transplantation. Eur J Cardiothorac Surg 1991;5:94-7.

2. Demertzis S, Haverich A, Ziemer G, et al. Successful lung transplantation for posttraumatic adult respiratory distress syndrome after extracorporeal membrane oxygenation support. J Heart Lung Transplant 1992;11:1005-7.

3. Todd EM, Roy SB, Hashimi AS, et al. Extracorporeal membrane oxygenation as a bridge to lung transplantation: A single-center experience in the present era. J Thorac Cardiovasc Surg 2017;154:1798-809.

4. Biscotti M, Gannon WD, Agerstrand C, et al. Awake Extracorporeal Membrane Oxygenation as Bridge to Lung Transplantation: A 9-Year Experience. Ann Thorac Surg 2017;104:412-9.

5. Ius F, Natanov R, Salman J, et al. Extracorporeal membrane oxygenation as bridge to lung transplantation may not impact overall mortality risk after transplantation: results from a 7-year single-centre experience. Eur J Cardiothorac Surg 2018. [Epub ahead of Print].

6. Hoetzenecker K, Donahoe L, Yeung JC, et al. Extracorporeal life support as a bridge to lung transplantation-experience of a high-volume transplant center. J Thorac Cardiovasc Surg 2018;155:1316-28.e1.

7. Hakim AH, Ahmad U, McCurry KR, et al. Contemporary Outcomes of Extracorporeal Membrane Oxygenation Used as Bridge to Lung Transplantation. Ann Thorac Surg 2018. [Epub ahead of print].

8. Bermudez CA, Shiose A, Esper SA, et al. Outcomes of
Intraoperative Venoarterial Extracorporeal Membrane Oxygenation Versus Cardiopulmonary Bypass During Lung Transplantation. Ann Thorac Surg 2014;98:193642; discussion 1942-3.

9. Biscotti M, Yang J, Sonett J, et al. Comparison of extracorporeal membrane versus cardiopulmonary bypass for lung transplantation. J Thorac Cardiovasc Surg 2014;148:2410-5.

10. Machuca TN, Collaud S, Mercier O, et al. Outcomes of intraoperative extracorporeal membrane oxygenation versus cardiopulmonary bypass for lung transplantation. J Thorac Cardiovasc Surg 2015;149:1152-7.

11. Hoechter DJ, von Dossow V, Winter H, et al. The Munich Lung Transplant Group: Intraoperative Extracorporeal Circulation in Lung Transplantation. Thorac Cardiovasc Surg 2015;63:706-14.

12. Ius F, Sommer W, Tudorache I, et al. Five-year experience with intraoperative extracorporeal membrane oxygenation in lung transplantation: Indications and midterm results. J Heart Lung Transplant 2016;35:49-58.

13. Pettenuzzo T, Faggi G, Di Gregorio G, et al. Blood Products Transfusion and Mid-Term Outcomes of Lung Transplanted Patients Under Extracorporeal Membrane Oxygenation Support. Prog Transplant 2018. [Epub ahead of print].

14. Hoetzenecker K, Schwarz S, Muckenhuber M, et al. Intraoperative extracorporeal membrane oxygenation and the possibility of postoperative prolongation improve survival in bilateral lung transplantation. J Thorac Cardiovasc Surg 2018;155:2193-206.e3.

15. Hartwig MG, Walczak R, Lin SS, et al. Improved Survival but Marginal Allograft Function in Patients Treated With Extracorporeal Membrane Oxygenation After Lung Transplantation. Ann Thorac Surg 2012;93:366-71.

16. Tudorache I, Sommer W, Kühn C, et al. Lung Transplantation for Severe Pulmonary HypertensionAwake Extracorporeal Membrane Oxygenation for Postoperative Left Ventricular Remodeling. Transplantation 2015;99:451-8.

17. Salman J, Ius F, Sommer W, et al. Mid-term results of bilateral lung transplant with postoperatively extended intraoperative extracorporeal membrane oxygenation for severe pulmonary hypertension. Eur J Cardiothorac Surg 2017;52:163-70.

18. Moser B, Jaksch P, Taghavi S, et al. Lung transplantation for idiopathic pulmonary arterial hypertension on intraoperative and postoperatively prolonged extracorporeal membrane oxygenation provides optimally 
controlled reperfusion and excellent outcome. Eur J Cardiothorac Surg 2018;53:178-85.

19. Loor G, Simpson L, Parulekar A. Bridging to lung transplantation with extracorporeal circulatory support: when or when not? J Thorac Dis 2017;9:3352-61.

20. Rajagopal K, Hoeper MM. State of the Art: Bridging to lung transplantation using artificial organ support technologies. J Heart Lung Transplant 2016;35:1385-98.

21. Schaheen LW, D'Cunha J. Extracorporeal membrane oxygenation in lung transplantation: No longer a fourletter word. J Thorac Cardiovasc Surg 2018;155:2191-2.

22. Reeb J, Olland A, Renaud S, et al. Vascular access for extracorporeal life support: tips and tricks. J Thorac Dis 2016;8:S353-63.

23. Fernandez R, DeCamp M, Bharat A. A novel strategy for cardiopulmonary support during lung transplantation. J Thorac Dis 2018;10:E142-4.

24. Diaz-Guzman E, Sharma NS, Wille K, et al. Use of a novel pulmonary artery cannula to provide extracorporeal membrane oxygenation as a bridge to lung transplantation. J Heart Lung Transplant 2016;35:1051-3.

25. Bermudez CA, Zaldonis D, Fan MH, et al. Prolonged Use of the Hemolung Respiratory Assist System as a Bridge to Redo Lung Transplantation. Ann Thorac Surg 2015;100:2330-3.

26. Fischer $\mathrm{S}$, Simon AR, Welte $T$, et al. Bridge to lung transplantation with the novel pumpless interventional lung assist device NovaLung. J Thorac Cardiovasc Surg 2006;131:719-23.

27. Strueber M, Hoeper MM, Fischer S, et al. Bridge to thoracic organ transplantation in patients with pulmonary artery hypertension using a pumpless lung assist device. Am J Transplant 2009;9:853-7.

28. Camboni D, Akay B, Pohlmann JR, et al. Veno-venous extracorporeal membrane oxygenation with interatrial shunting: a novel approach to lung transplantation for patients in right ventricular failure. J Thorac Cardiovasc Surg 2011;141:537-42.

29. Hartwig MG, Appel JZ III, Cantu E III, et al. Improved Results Treating Lung Allograft Failure With Venovenous Extracorporeal Membrane Oxygenation. Ann Thorac Surg 2005;80:1872-9; discussion 1879-80.

30. Chicotka S, Pedroso FE, Agerstrand CL, et al. Increasing Opportunity for Lung Transplant in Interstitial Lung Disease with Pulmonary Hypertension. Ann Thorac Surg 2018. [Epub ahead of print].

31. Glorion M, Mercier O, Mitilian D, et al. Central versus peripheral cannulation of extracorporeal membrane oxygenation support during double lung transplant for pulmonary hypertension. Eur J Cardiothorac Surg 2018. [Epub ahead of print].

32. Ius F, Sommer W, Tudorache I, et al. Veno-veno-arterial extracorporeal membrane oxygenation for respiratory failure with severe haemodynamic impairment: technique and early outcomes. Interact Cardiovasc Thorac Surg 2015;20:761-7.

33. Wong JK, Melvin AL, Joshi DJ, et al. CannulationRelated Complications on Veno-Arterial Extracorporeal Membrane Oxygenation: Prevalence and Effect on Mortality. Artif Organs 2017;41:827-34.

34. Bisdas T, Beutel G, Warnecke G, et al. Vascular complications in patients undergoing femoral cannulation for extracorporeal membrane oxygenation support. Ann Thorac Surg 2011;92:626-31.

35. Bermudez CA, Rocha RV, Zaldonis D, et al. Extracorporeal membrane oxygenation as a bridge to lung transplant: midterm outcomes. Ann Thorac Surg 2011;92:1226-31.

36. Fuehner T, Kuehn C, Hadem J, et al. Extracorporeal membrane oxygenation in awake patients as bridge to lung transplantation. Am J Respir Crit Care Med 2012;185:763-8.

37. Lang G, Taghavi S, Aigner C, et al. Primary lung transplantation after bridge with extracorporeal membrane oxygenation: a plea for a shift in our paradigms for indications. Transplantation 2012;93:729-36.

38. Javidfar J, Brodie D, Iribarne A, et al. Extracorporeal membrane oxygenation as a bridge to lung transplantation and recovery. J Thorac Cardiovasc Surg 2012;144:716-21.

39. Toyoda Y, Bhama JK, Shigemura N, et al. Efficacy of extracorporeal membrane oxygenation as a bridge to lung transplantation. J Thorac Cardiovasc Surg 2013;145:1065-70.

40. Lafarge $M$, Mordant $P$, Thabut G, et al. Experience of extracorporeal membrane oxygenation as a bridge to lung transplantation in France. J Heart Lung Transplant 2013;32:905-13.

41. Dellgren G, Riise GC, Swärd K, et al. Extracorporeal membrane oxygenation as a bridge to lung transplantation: a long-term study. Eur J Cardiothorac Surg 2015;47:95-100.

42. Yeo HJ, Lee S, Yoon SH, et al. Extracorporeal Life Support as a Bridge to Lung Transplantation in Patients with Acute Respiratory Failure. Transplant Proc 2017;49:1430-5.

43. Bittner HB, Lehmann S, Rastan A, et al. Outcome of 
extracorporeal membrane oxygenation as bridge to lung transplantation and graft recovery. Ann Thorac Surg 2012;94:942-9.

44. Schmidt F, Sasse M, Boehne M, et al. Concept of "awake venovenous extracorporeal membrane oxygenation" in pediatric patients awaiting lung transplantation. Pediatr Transplant 2013;17:224-30.

45. Lang G, Kim D, Aigner C, et al. Awake extracorporeal membrane oxygenation bridging for pulmonary retransplantation provides comparable results to elective retransplantation. J Heart Lung Transplant 2014;33:1264-72.

46. Hayanga AJ, Aboagye J, Esper S, et al. Extracorporeal membrane oxygenation as a bridge to lung transplantation in the United States: an evolving strategy in the management of rapidly advancing pulmonary disease. J Thorac Cardiovasc Surg 2015;149:291-6

47. Hayes D Jr, Tobias JD, Tumin D. Center Volume and Extracorporeal Membrane Oxygenation Support at Lung Transplantation in the Lung Allocation Score Era. Am J Respir Crit Care Med 2016;194:317-26.

48. Schechter MA, Ganapathi AM, Englum BR, et al. Spontaneously Breathing Extracorporeal Membrane Oxygenation Support Provides the Optimal Bridge to Lung Transplantation. Transplantation 2016;100:2699-704.

49. Tsiouris A, Budev MM, Yun JJ. Extracorporeal Membrane Oxygenation as a Bridge to Lung Transplantation in the United States: A Multicenter Survey. ASAIO J 2017. [Epub ahead of print].

50. Turner DA, Cheifetz IM, Rehder KJ, et al. Active rehabilitation and physical therapy during extracorporeal membrane oxygenation while awaiting lung transplantation: a practical approach. Crit Care Med 2011;39:2593-8.

51. Rahimi RA, Skrzat J, Reddy DRS, et al. Physical Rehabilitation of Patients in the Intensive Care Unit Requiring Extracorporeal Membrane Oxygenation: A Small Case Series. Phys Ther 2013;93:248-55.

52. Hayes K, Hodgson CL, Pellegrino VA, et al. Physical Function in Subjects Requiring Extracorporeal Membrane Oxygenation Before or After Lung Transplantation. Respir Care 2018;63:194-202.

53. Kolaitis NA, Soong A, Shrestha P, et al. Improvement in patient-reported outcomes after lung transplantation is not impacted by the use of extracorporeal membrane oxygenation as a bridge to transplantation. J Thorac Cardiovasc Surg 2018;156:440-8.e2.

54. Hayanga AJ, Du AL, Joubert K, et al. Mechanical
Ventilation and Extracorporeal Membrane Oxygenation as a Bridging Strategy to Lung Transplantation: Significant Gains in Survival. Am J Transplant 2018;18:125-35.

55. Ius F, Kuehn C, Tudorache I, et al. Lung transplantation on cardiopulmonary support: Venoarterial extracorporeal membrane oxygenation outperformed cardiopulmonary bypass. J Thorac Cardiovasc Surg 2012;144:1510-6.

56. Aigner $\mathrm{C}, \mathrm{Wisser} W$, Taghavi S, et al. Institutional experience with extracorporeal membrane oxygenation in lung transplantation. Eur J Cardiothorac Surg 2007;31:468-73; discussion 473-4.

57. Yu WS, Paik HC, Haam SJ, et al. Transition to routine use of venoarterial extracorporeal oxygenation during lung transplantation could improve early outcomes. J Thorac Dis 2016;8:1712-20.

58. Cosgun T, Tomaszek S, Opitz I, et al. Single-center experience with intraoperative extracorporeal membrane oxygenation use in lung transplantation. Int J Artif Organs 2017. [Epub ahead of print].

59. Magouliotis DE, Tasiopoulou VS, Svokos AA, et al. Extracorporeal membrane oxygenation versus cardiopulmonary bypass during lung transplantation: a meta-analysis. Gen Thorac Cardiovasc Surg 2018;66:38-47.

60. Hoechter DJ, Shen YM, Kammerer T, et al. Extracorporeal Circulation During Lung Transplantation Procedures: A meta-Analysis. ASAIO J 2017;63:551-61.

61. Ribeiro Neto ML, Budev M, Culver DA, et al. Venous Thromboembolism After Adult Lung Transplantation: A Frequent Event Associated With Lower Survival. Transplantation 2018;102:681-7.

62. Narm KS, Lee S, Suh JW, et al. Risk Factor Analysis for Intraoperative Extracorporeal Membrane Oxygenation Weaning Failure After Lung Transplantation. Ann Thorac Surg 2018;105:242-8.

63. Vlasselaers D, Verleden GM, Meyns B, et al. Femoral Venoarterial Extracorporeal Membrane Oxygenation for Severe Reimplantation Response After Lung Transplantation. Chest 2000;118:559-61.

64. Meyers BF, Sundt TM III, Henry S, et al. Selective use of extracorporeal membrane oxygenation is warranted after lung transplantation. J Thorac Cardiovasc Surg 2000;120:20-6.

65. Dahlberg PS, Prekker ME, Herrington CS, et al. MediumTerm Results of Extracorporeal Membrane Oxygenation for Severe Acute Lung Injury After Lung Transplantation. J Heart Lung Transplant 2004;23:979-84.

66. Oto T, Rosenfeldt F, Rowland M, et al. Extracorporeal Membrane Oxygenation After Lung Transplantation: 
Evolving Technique Improves Outcomes. Ann Thorac Surg 2004;78:1230-5.

67. Mason DP, Boffa DJ, Murthy SC, et al. Extended use of extracorporeal membrane oxygenation after lung transplantation. J Thorac Cardiovasc Surg 2006;132:954-60.

68. Fischer S, Bohn D, Rycus P, et al. Extracorporeal membrane Oxygenation for Primary Graft Dysfunction After Lung Transplantation: Analysis of the Extracorporeal Life Support Organization (ELSO) Registry. J Heart Lung Transplant 2007;26:472-7.

69. Bermudez CA, Adusumilli PS, McCurry KR, et al. Extracorporeal Membrane Oxygenation for Primary Graft Dysfunction After Lung Transplantation: Long-Term Survival. Ann Thorac Surg 2009;87:854-60.

70. Marasco SF, Vale M, Preovolos A, et al. Institution of extracorporeal membrane oxygenation late after lung transplantation-a futile exercise? Clin Transplant

Cite this article as: Ius F, Tudorache I, Warnecke G. Extracorporeal support, during and after lung transplantation: the history of an idea. J Thorac Dis 2018;10(8):5131-5148. doi: $10.21037 /$ jtd.2018.07.43
2012;26:E71-7.

71. Mulvihill MS, Yerokun BA, Davis RP, et al. Extracorporeal membrane oxygenation following lung transplantation: indications and survival. J Heart Lung Transplant 2017;37:259-67.

72. Snell GI, Yusen RD, Weill D, et al. Report of the ISHLT Working Group on Primary Lung Graft Dysfunction, part I: Definition and grading-A 2016 Consensus Group statement of the International Society for Heart and Lung Transplantation. J Heart Lung Transplant 2017;36:1097-103.

73. Shah RJ, Diamond JM. Primary Graft Dysfunction (PGD) Following Lung Transplantation. Semin Respir Crit Care Med 2018;39:148-54.

74. Porteous MK, Ky B, Kirkpatrick JN, et al. Diastolic Dysfunction Increases the Risk of Primary Graft Dysfunction after Lung Transplant. Am J Respir Crit Care Med 2016;193:1392-400. 